DESY 96-058

ISSN 0418-9833

April 1996

\title{
AROMA 2.2 - A Monte Carlo Generator for Heavy Flavour Events in ep Collisions
}

\author{
G. Ingelman ${ }^{a, b}$, J. Rathsman ${ }^{b}$, G.A. Schuler ${ }^{c}$ \\ ingelman@desy.deｒathsman@tsl.uu.serschulerg@afsmail.cern.ch
}

${ }^{a}$ Deutsches Elektronen-Synchrotron DESY, Notkestrasse 85, D-22603 Hamburg, FRG

${ }^{b}$ Dept. of Radiation Sciences, Uppsala University, Box 535, S-751 21 Uppsala, Sweden

${ }^{c}$ Theory Division, CERN, CH-1211 Geneva 23, Switzerland

\begin{abstract}
A program to simulate the production of heavy quarks through the boson-gluon fusion process in $e^{ \pm} p$ collisions is presented. The full electroweak structure of the electron-gluon interaction is taken into account as well as the masses of the produced heavy quarks. Higher order QCD radiation is treated using initial and final state parton showers, and hadronization is performed using the Lund string model. Physics and programming aspects are described in this manual.
\end{abstract}

\section{Physics of included processes}

The production of heavy quarks in ep collisions is dominated by boson-gluon fusion (BGF) into a heavy quark-antiquark pair

$$
V(q)+g(p) \rightarrow Q_{f}\left(p_{f}\right)+\bar{Q}_{f^{\prime}}\left(p_{f^{\prime}}\right),
$$

occuring as $\mathcal{O}\left(\alpha_{s} \alpha^{2}\right)$ parton level subprocess in the ep scattering process

$$
e^{ \pm}\left(l_{e}\right)+p(P) \rightarrow l^{\prime}\left(l^{\prime}\right)+Q_{f}\left(p_{f}\right)+\bar{Q}_{f^{\prime}}\left(p_{f^{\prime}}\right)+X .
$$

Here the symbols in brackets denote the corresponding four-momenta. In the charged current $(\mathrm{CC})$ case $V$ is the $W^{ \pm}$-boson whereas in the neutral current (NC) case it corresponds to $\gamma / Z^{0}$ exchange and the produced quark and antiquark have the same flavour $f$. Beside the normal DIS variables defined by

$$
Q^{2} \equiv-q^{2}=-\left(l_{e}-l^{\prime}\right)^{2} \quad, \quad W^{2} \equiv(P+q)^{2} \quad, \quad x \equiv \frac{Q^{2}}{2 P \cdot q} \quad, \quad y \equiv \frac{P \cdot q}{P \cdot l_{e}},
$$

out of which only two are independent, three additional independent variables are needed to completely specify the process in eq. (2). These are here taken as the momentum fraction $x_{g}$ of the gluon relative to the proton, i.e. $p=x_{g} P$, the variable

$$
z=\frac{P \cdot p_{f}}{P \cdot q}
$$

\footnotetext{
${ }^{1}$ Heisenberg fellow
} 
and the azimuthal (around the boson axis) angle $\Phi$ between the lepton and hadron planes

$$
\cos \Phi=\frac{\left(\vec{p} \times \overrightarrow{l_{e}}\right) \cdot\left(\vec{p} \times \overrightarrow{p_{f}}\right)}{\left|\vec{p} \times \overrightarrow{l_{e}}\right|\left|\vec{p} \times \overrightarrow{p_{f}}\right|}
$$

measured in the boson-gluon CM frame, i.e. $\vec{p}_{f}+\vec{p}_{f^{\prime}}=0$. The gluon momentum fraction is related to the usual Bjorken- $x$ variable by

$$
x_{g}=x+\frac{\hat{s}}{y s} \geq x .
$$

The variable $z$ is related to the angle of the $Q \bar{Q}^{\prime}$-axis with respect to the boson-gluon axis in this subsystem cms and gives the heavy quark transverse momentum through the relation $p_{\perp}^{2}=\hat{s} z(1-z)+z\left(m_{f^{\prime}}^{2}-m_{f}^{2}\right)-m_{f}^{2}$. As usual, $\hat{s}$ is taken to denote the invariant mass square of the $Q \bar{Q}^{\prime}$-subsystem, i.e. $\hat{s}=\left(p_{f}+p_{f^{\prime}}\right)^{2}$.

The cross section for process (2) is then given by

$$
\sigma\left(e^{ \pm} p \rightarrow Q \bar{Q}^{\prime} X\right)=\int d y \int d Q^{2} \int d x_{g} \int d z \int d \Phi g\left(x_{g}, M_{g}^{2}\right) h\left(y, Q^{2}, x_{g}, z, \Phi\right)
$$

which is a convolution of the gluon density $g\left(x_{g}, M_{g}^{2}\right)$ and a QCD part $h$ for the subprocess. The latter has been calculated in ref. [1] taking proper account of the heavy quark masses and the complete electroweak structure for both charged and neutral current processes including the $\gamma-Z^{0}$ interference and allowing for arbitrary longitudinally polarization of the $e^{ \pm}$beam. Both the deep inelastic and photoproduction $\left(Q^{2} \rightarrow 0\right)$ region is covered.

For the numerical evaluation [1, 2] of this cross section the electroweak parameters are taken from Lepto 6.5 [3]. The Kobayashi-Maskawa matrix elements have the default values of LEPTO 6.5 and the quark masses the default values of JETSET 7.4 [4, in particular $m_{c}=1.35 \mathrm{GeV}$ and $m_{b}=5 \mathrm{GeV}$. All these default values can be changed by the user. The mass scales for the gluon density and for $\alpha_{s}, M_{g}$ and $M_{s}$, are taken to be $M_{g}=M_{s}=\sqrt{\hat{s}}$; the number of flavours, $N_{f}$, and $\Lambda_{Q C D}$ used in the first order expression for $\alpha_{s}$ are taken from JETSET which means that $\Lambda_{Q C D}$ is taken from the parton densities. A selection of parametrizations for the gluon density in the proton are provided as alternatives (cf. LST(15) [3]). The numerical uncertainties in the cross sections are discussed in [2].

The next-to-leading order (NLO) QCD corrections to the inclusive heavy quark distributions (rapidity and $p_{\perp}$ ) have been calculated for photoproduction $\left(Q^{2} \ll \Lambda^{2}\right)$ [5] and recently also for deep-inelastic scattering (DIS), i.e. $Q^{2} \gg \Lambda^{2}$ [6]. In the case of photoproduction, the $\mathcal{O}\left(\alpha_{s}^{2}\right)$ corrected distributions are found to be similar in shape to the leading-order ones, i.e. they can be described by an approximately constant $K$-factor. It is therefore expected that the distributions from AROMA describe the shapes of inclusive heavy quark distributions reasonably well, whereas the absolute normalization is underestimated [5]. In contrast, the $K$-factor of DIS is strongly kinematics dependent and therefore not easily taken into account. In addition, the available NLO result only applies for inclusive quantities and not for the exclusive final state needed for MC simulation.

For a proper description of the complete event topology higher order corrections are important. Gluon bremsstrahlung off the heavy quark and antiquark can be approximately taken into account by the use of parton shower (PS) simulation algorithms. From the experience of jets in $e^{+} e^{-}$annihilation one expects this approach, which includes the leading logarithm contribution of higher corrections, to provide a better description of 
detailed jet properties, such as hardness and width, as compared to exact next-to-leading order matrix elements. Such a shower algorithm [- 1 is therefore applied, but one should note that the rate of clearly separable additional jets need not be quite correct.

Production of heavy quarks in lowest order, eq. (1), is characterized by a pair of heavy quarks that are essentially back-to-back in the boson-proton CMS and with small overall transverse momentum $p_{\perp}(Q \bar{Q})$. Small deviations from this can arise from a non-vanishing primordial $k_{\perp}$ of the incoming gluon, which is here generated according to a Gaussian distribution. Larger deviations from this topology may arise through parton radiation from the gluon entering the fusion process. Such initial state radiation also affects the inclusive heavy quark spectra, which in NLO accuracy follow rather closely those at the Born level (at least at low $Q^{2}$ ). This implies constraints on the initial radiation and therefore one does not expect large effects. Initial state parton showers are implemented by suitably modifying the algorithm of ref. [7], in particular allowing for a smooth $Q^{2} \rightarrow 0$ limit.

In ep scattering heavy quarks can also be produced through (i) mixing in the leading order charged current process $e+q \rightarrow \nu+Q$ [2], (ii) the scattering on intrinsic charm quarks in the proton [8], (iii) resolved photon contributions leading to hadronic production processes of heavy flavours (e.g. $g g \rightarrow Q \bar{Q}$ ) and (iv) diffractive heavy flavour excitation 9]. These processes, which are not included in AROMA, are expected to have much smaller cross sections than the BGF process in the HERA energy range.

The Aroma program is also applicable to the case of light flavours. However, without the heavy quark mass as a cutoff against the divergences in the matrix element it is then necessary to apply a lower cut on the $p_{\perp}$ of the produced quarks in the boson-gluon fusion cms. The program thus allows to set a $p_{\perp}$-cutoff, which may also be applied in heavy quark generation.

\section{The Monte Carlo implementation}

The Monte Carlo simulation model is based on the following main ingredients: (i) The complete matrix elements [1] to order $\alpha^{2} \alpha_{s}$ for the boson-gluon fusion process, eq. (7), (including the masses of the produced heavy quarks and the full electroweak structure of the interaction), (ii) gluon emission from the $Q \bar{Q}^{\prime}$ system in a parton shower approach [4, (iii) initial state parton shower, (iv) possible soft colour interactions as a mechanism for rapidity gaps [10], (v) hadronization with the Lund string model [11, [1] and heavy flavour decay.

The importance sampling method in Divonne [12] is used to generate phase space points according to the differential cross-section formula in eq. (7). From the phase space point $\left(y, Q^{2}, x_{g}, z, \Phi\right)$, the four-momenta of all partons (particles) are calculated.

Similarly to the $q \bar{q}$ pair produced in $e^{+} e^{-}$annihilation the heavy $Q \bar{Q}^{\prime}$ pair can emit bremsstrahlung gluons (some of which may split perturbatively into gluon or $q \bar{q}$ pairs) thereby creating a cascade, or shower, at the parton level. To simulate this the model for $e^{+} e^{-}[4]$ is applied. In doing so an uncertainty arises as to what scale to use for the maximum off-shellness of the heavy quark and antiquark that initiate the shower. Different options are provided, see $\operatorname{LSTHFL}(7)$, with the default chosen as $\left(m_{\perp Q}+m_{\perp \bar{Q}^{\prime}}\right)^{2}$ since this measures the momentum transfer in similarity with the case in the $g g \rightarrow$ 
$Q \bar{Q}$ process in hadron collisions. To account for parton radiation from the incoming gluon, the initial state parton shower [7] in LEPTO has been incorporated using the scale $\min \left(\mu^{2}, W^{2}-\hat{s}, \hat{s}+Q^{2} / 2\right)$ where $\mu^{2}$ is set by LSTHFL(9) (default is $\left.\left(m_{\perp Q}+m_{\perp \bar{Q}^{\prime}}\right)^{2}\right)$.

The multiparton final state is then supplemented by the proton remnant. In case of an incoming gluon, the remnant contains the three valence quarks. The energy-momentum of the remnant is divided and a small relative transverse momentum introduced when this system is split [13, 3] into a quark and a diquark. Together with the produced heavy antiquark and quark, respectively, these spectator partons form two separate colour triplet strings. Before hadronising these strings with JETSET 7.4 [4] extra soft colour interactions may be taken into account? which is regulated by LST(34). This results in a Monte Carlo model for heavy flavour production in $e p$ collisions that generates complete events with the full information on both the parton and the hadron level.

\section{Description of program components}

The program is written in FORTRAN 77 and consists of a set of subroutines that form an add-on package to Lepto 6.5 [3]. In addition, Jetset 7.4 and Pythia 5.7 四 and the Divonne program [12], which are all available via CERnLiB, are used. The user has to ensure that the Aroma routines are loaded such that they replace some routines in LEPTO with their modified versions, i.e. normally Aroma should be loaded first. All subroutine and common-block names start with HF to avoid name clashes. Exceptions are: AROMA and AROINI which the user calls; DFUN and DVNOPT which replaces subroutines in LEPTO 6.5 and the block data ARODAT.

The heavy flavour generation is made by calling AROMA. The parameters, cuts and switches for heavy flavour generation are in arrays PARHFL, CUTHFL and LSTHFL of common HFDATA described below.

\subsection{Subroutines and functions}

The following routines are called by the user:

\section{SUBROUTINE AROINI(LFILE,LEPIN,PLZ,PPZ,INTER)}

Purpose: $\quad$ to initialize the event generation procedure, see also [3].

Arguments:

LFILE : $\quad=0$ normally when using Aroma.

LEPIN : $\quad$ type of lepton in JETSET 7.4 code, i.e. $11=e^{-},-11=e^{+}$.

PLZ, PPZ : momentum $(\mathrm{GeV} / \mathrm{c})$ for incoming lepton and nucleon, respectively, along the z-axis (if both non-zero, i.e. colliding beams, they must have opposite signs).

INTER : $\quad$ type of interaction to be simulated.

$=1$ : electromagnetic $(\mathrm{EM})$, i.e. $\gamma$ exchange.

$=2$ : weak charged current $(\mathrm{CC})$, i.e. $W^{ \pm}$exchange.

$=3$ : weak neutral current, i.e. $Z^{0}$ exchange.

\footnotetext{
${ }^{2}$ This will give some production of $q \bar{q}$ resonances for sufficiently small masses of the $q \bar{q}$ system. However, the treatment is rather crude and only $J / \psi$ and $\Upsilon$ are produced for $c \bar{c}$ and $b \bar{b}$ respectively.
} 
$=4$ : neutral current $(\mathrm{NC})$, i.e. $\gamma / Z^{0}$ exchange.

\section{SUBROUTINE AROMA}

Purpose: $\quad$ to administer the generation of one event of the kind specified by the last AROINI call.

Remark: If the error flag LST(21) is non-zero then the event should be rejected (should only occur rarely).

\section{SUBROUTINE LFRAME(IFRAME,IPHI)}

Purpose: $\quad$ to transform the event between different frames, see [3].

In the following list all subroutines (S) and functions (F) are briefly described. The order is as they appear in the code and reflects the flow in the program. Further details about their purpose and procedures used are given by comments in the code.

Routine Purpose

ARODAT Block data to give default values to all switches and parameters.

AROINI (S) To initialize program, see above.

HFINIT (S) Initiate constants, calculate integrated cross section, set-up grid for event generation. Called by AROINI.

AROMA (S) To generate an event, see above.

HFLGEN (S) Administer heavy flavour generation based on exact matrix elements, called by AROMA.

DVNOPT (S) Substitutes block data for Divonne.

DFUN (F) Integrand for Divonne, calls HFFUNC.

HFFUNC (F) Differential cross section summed over flavours.

HFVECT (S) Transforms XX(1), .., XX(4) to $y, Q^{2}, x_{g}, z$.

HFCHCK (S) Check if $y, Q^{2}, x_{g}$ and $z$ are within bounds for given flavours.

HFTOTD (F) Differential cross section for given flavours.

HFVCT1 (S) Variable mapping for $\gamma$-exchange, called by HFVECT.

HFVCT2 (S) Variable mapping for $W$ - and $Z$-exchange, called by HFVECT.

HFMEPS (S) Modified version of LMEPS in LEPTO.

HFSCAL (S) Modified version of LSCALE in LEPTO.

HFSSPA (S) Modified version of LYSSPA in LEPTO.

HFANGL (F) Modified version of ULANGL in JETSET.

HFDBRB (S) Modified version of LUROBO in JETSET.

The following subroutines in Divonne 4 are used: PARTN, INTGRL, RANGEN.

\subsection{Common blocks}

The common blocks intended for communication with the program are HFDATA and LEPTOU. Common LUJETS in Jetset 7.4 四 is used to store the event record and is therefore essential. All variables are given sensible default values in the block data ARODAT, as shown by $(\mathrm{D}=\ldots)$ below. Overwriting the default values should be made before calling AROINI. 


\section{COMMON /HFDATA/ PARHFL(10),CUTHFL(8),LSTHFL(10)}

Purpose: $\quad$ contains input switches and input parameters to specify physics, kinematic cuts and numerical procedures, as well as output flags. Note that PARHFL and CUTHFL are in double precision.

PARHFL(1) : $\quad$ total cross section in $p b$ for heavy flavour production. Calculated in the initialization taking the hard cuts in CUTHFL into account.

PARHFL(2) : $\quad(\mathrm{D}=0.2)$ precision parameter SPRDMX for call to PARTN in the DIVONNE program; smaller values give finer partitioning.

PARHFL(3): $\quad(\mathrm{D}=1000$.) parameter NPT for call to INTGRL in Divonne. Specifies maximum function evaluations in each subregion for integrating total heavy flavour cross section.

PARHFL $(4): \quad\left(\mathrm{D}=0 . \mathrm{GeV}^{2}\right)$ defines the minimum $p_{\perp}^{2}$ of the quarks in the boson-gluon cms, must be used when producing light quarks.

PARHFL(5): fraction of phase space points generated within the 'hard' cuts given in CUTHFL (see below) that are accepted by the 'soft' cuts in CUT (see common LEPTOU in [3]) when generating the complete events.

PARHFL(6) : cross-section in $p b$ for the generated event sample. Given by the product of PARHFL(1) and PARHFL(5), thus taking both hard and soft cuts into account. To be used at the end of event generation (updated for each event) to get absolute cross section normalisation of generated event sample.

PARHFL(7-10) : not used

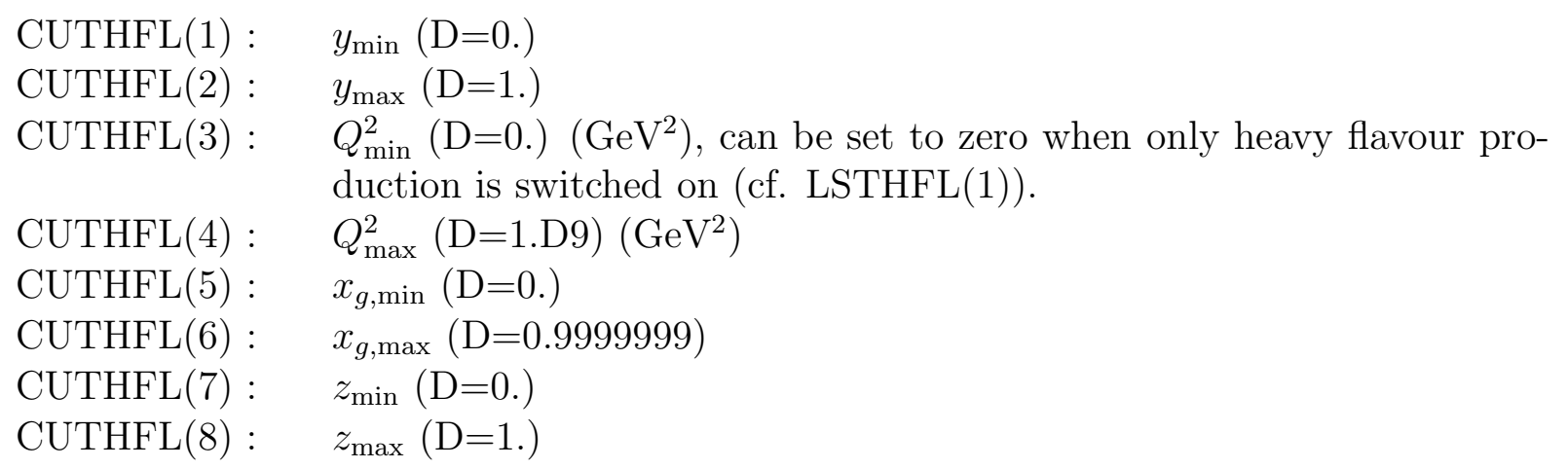

Remark: These 'hard' cuts are on the basic variables in the heavy flavour matrix element and can therefore be efficiently applied to limit the region of phase space. (Kinematic phase space limits are explicitly calculated and applied if stronger than these cuts.) In contrast, the 'soft' cuts in CUT (common LEPTOU in [3]) are used to reject chosen points or partly generated events, resulting in less efficiently applied cuts. See PARHFL(5), PARHFL(6) above.

LSTHFL(1): $\quad(\mathrm{D}=2)$ regulates heavy flavour generation.

$=0$ : DIS events, no heavy flavour production.

$=1$ : DIS events and heavy flavour production.

$=2$ : only heavy flavour production.

Remark: When 'normal' LEPTo events and heavy flavour events are mixed in the same run, the total cross section is then the sum of the normal one (PARL(23) or PARL(24)) and the heavy flavour one (PARHFL(6)). To avoid double counting precautions have been taken in AROINI by 
setting switches such that the same flavours should not be produced in other processes; i.e. boson-gluon fusion process with massless matrix elements in LEPTo (LST(13)), through parton showers (MSTJ(45), IPY(8)) and via sea quark density parametrizations (LST(12)).

LSTHFL(2), LSTHFL(3) : $(\mathrm{D}=4,5)$ lightest and heaviest flavour to be produced in NC heavy flavour production, can be set between 1 and 6 for $d, u, s, c, b, t$.

LSTHFL(4): $\quad(\mathrm{D}=4)$ specifies process simulated

$=1$ : pure $\gamma$ contribution.

$=2$ : $W$ exchange.

$=3$ : pure $Z$ contribution.

=4: full $\mathrm{NC}$ contribution $(\gamma+\gamma / Z+Z)$.

$\operatorname{LSTHFL(5):~} \quad$ internal, $>0$ for $e^{+},<0$ for $e^{-}$.

LSTHFL(6) : $\quad(\mathrm{D}=11)$ regulates which flavour combinations to be included in CC interactions. Should be set to $I_{c s}+10 I_{c b}+100 I_{t s}+1000 I_{t b}$, where $I_{c s}$ etc. is 0 to exclude (1 to include) $c \bar{s}$ (or $\bar{c} s$ ) etc.

$\operatorname{LSTHFL}(7): \quad(\mathrm{D}=4)$ regulates the scale in the final state parton showers in $Q \bar{Q}$ system. Value is multiplied by $\operatorname{PYPAR}(25)$.

$=1: Q^{2}$,

$=2: W^{2}$,

$=3$ : $\hat{s}$, i.e. same treatment as for $q \bar{q}$-event in LEPTO

$=4:\left(m_{\perp Q}+m_{\perp \bar{Q}}\right)^{2}$,

$=5: m_{\perp Q}^{2}+m_{\perp \bar{Q}}^{2}$, where $m_{\perp Q}$ is the transverse mass of the heavy quark.

LSTHFL(8): $\quad(\mathrm{D}=2)$ simulation of QCD effects in hadronic final state, see [3]

$=1$ : no parton showers

$=2$ : both initial and final state parton showers

$=3$ : initial state parton shower

=4: final state parton shower

LSTHFL $(9)$ : $\quad(\mathrm{D}=4)$ choice of scale, i.e. maximum parton offshellness, in QCD initial state cascade. Value is multiplied by $\operatorname{PYPAR}(26)$.

$=1: Q^{2}$,

$=2: W^{2}$,

$=3: \hat{s}$,

$=4:\left(m_{\perp Q}+m_{\perp \bar{Q}}\right)^{2}$,

$=5: m_{\perp Q}^{2}+m_{\perp \bar{Q}}^{2}$,

$=6$ : Same treatment as for $q \bar{q}$-event in LePTo.

Remark: For the initial state shower the scale used is $\min \left(\mu^{2}, W^{2}-\hat{s}, \hat{s}+Q^{2} / 2\right)$ where $\mu^{2}$ is the scale chosen above $\left(Q^{2}\right.$ should not be used for photoproduction).

COMMON /LEPTOU/ CUT(14),LST(40),PARL(30),X,Y,W2,Q2,U

Purpose: $\quad$ contains input switches and input parameters to specify physics, soft kinematic cuts and numerical procedures, as well as output flags and output variables.

Remark: only changes for AROMA are listed here, otherwise see [3].

$\operatorname{LST}(8)$ : set by AROINI corresponding to LSTHFL(8).

$\mathrm{LST}(17)=1$ : not usable with Aroma.

$\operatorname{LST}(24)=5$ : current event is heavy quark event from AromA.

LST(35) : $\quad$ there is no special sea quark treatment in the initial cascade in Aroma. 
COMMON /HFLVAR/ SHAT,ETA,Z,PHIQQ,PT2Q,TM2Q1,TM2Q2,ZJ,IFLQ,IFLQB, \& PHIFL(0:9,0:2),DBETAZ

Purpose: Variables at the parton level, values obtained from simulation of cross section. Note that DBETAZ is of type double precision.

SHAT,ETA,Z,PHIQQ : variables $\hat{s}, x_{g}, z$ and $\Phi$, see section 1 .

PT2Q : $\quad p_{\perp}^{2}$ for produced $Q$ (and $\bar{Q}$ ) in the boson-gluon cms, see section 1 .

TM2Q1,TM2Q2 : $m_{\perp}^{2}$ for produced $Q$ and $\bar{Q}$ in the boson-gluon cms.

COMMON /LFLMIX/ CABIBO $(4,4)$

Purpose: Contains the Cabbibo-Kobayashi-Maskawa matrix elements squared for flavour mixing, see [3].

COMMON /LUDAT2/ ...PMAS(500,4) ..

Purpose: Various parameters in JETSET [4].

PMAS(IFL,1) : mass for quark IFL $=1-6$ for $d, u, s, c, b, t$ used in the BGF matrix element

COMMON /LUJETS/ N,K(4000,5),P(4000,5),V(4000,5)

Purpose: Contains the record of the generated event, see [4].

Remark: The event record contains the full development of the event including a few extra, but inactive lines used for internal testing purposes.

The following common-blocks are mainly for internal use: HFBL1, HFBL2, HFJETS, HFJRDM, HFMACH, HFSNCO.

DIVONne common-blocks used: BNDLMT, SAMPLE, PRINT, QUADRE.

\section{Usage and Availability}

Aroma 2.2 should be loaded together with Divonne [12], Lepto 6.5 [3], Jetset 7.4 and Pythia 5.7 [4]. The program is a slave system, which the user must call from his own steering program. Information about the program, the source code and a demonstration job can be obtained from the authors or via the WWW on the Aroma homepage, http://www3.tsl.uu.se/thep/aroma/.

\section{References}

[1] G.A. Schuler, Nucl. Phys. B299 (1988) 21.

[2] G. Ingelman, G.A. Schuler, Z. Phys. C40 (1988) 299.

[3] G. Ingelman, A. Edin, J. Rathsman, LePto 6.5, program manual, DESY 96-057

[4] T. Sjöstrand, Pythia 5.7 and Jetset 7.4, CERN-TH.7112/93

T. Sjöstrand, Comp. Phys. Comm. 82 (1994) 74

[5] J. Smith and W.L. van Neerven, Nucl. Phys. B374 (1992) 36

R.K. Ellis and P. Nason, Nucl. Phys. B312 (1989) 551

[6] E. Laenen, S. Riemersma, J. Smith, W.L. van Neerven, Nucl. Phys. B392 (1993) 229 
[7] M. Bengtsson and T. Sjöstrand, Z. Phys. C37 (1988) 465.

[8] G. Ingelman, L. Jönsson, M. Nyberg, Phys. Rev. D47 (1993) 4872 and refs. therein

[9] P. Bruni, G. Ingelman, A. Solano, in Proc. 'Physics at HERA', Eds. W. Buchmueller, G. Ingelman, DESY Hamburg 1992, vol. 1, p. 363

[10] A. Edin, G. Ingelman, J. Rathsman, Phys. Lett. B366 (1996) 371

[11] B. Andersson, G. Gustafson, G. Ingelman, T. Sjöstrand, Phys. Rep. 97 (1983) 31

[12] J. Friedman, M. Wright, Divonne 4, CERN library program D151.

[13] B. Andersson, G. Gustafson, G. Ingelman, T. Sjöstrand, Z. Phys. C13 (1982) 361. 